ISSN 1997-5902

\title{
Potentiel invasif des espèces végétales exotiques ornementales des jardins de fleurs de la ville de Douala (Cameroun)
}

\author{
Mathurin TCHATAT¹, Samuel BOWONG², Christian Gaël Nembot Fomba3 , Siegfried Didier \\ DIBONG*(3,4,5) \\ 1. Institut de Recherche Agricole pour le Développement (IRAD), B.P. 2123 Yaoundé, Cameroun \\ 2. Département de Mathématique et Informatique, Faculté des Sciences, B.P. 812 Douala, Cameroun \\ 3. Département de Biologie des Organismes Végétaux, Faculté des Sciences, B.P. 812 Douala, Cameroun \\ 4. Département des Sciences Pharmaceutiques, Faculté de Médecine et des Sciences Pharmaceutiques, Université \\ de Douala, B.P. 2701 Douala, Cameroun \\ 5. Département d'Aquaculture, Institut des Sciences Halieutiques, B.P. 2701 Douala, Cameroun \\ Auteur de la correspondance: didierdibong@yahoo.fr
}

Original submitted in on 19th March 2014. Published online at www.m.elewa.org on $30^{\text {th June }} 2014$. http://dx.doi.org/10.4314/jab.v78i1.4

\section{RESUME}

Objectif : L'objectif de cette étude a été de Déterminer le potentiel invasif des espèces végétales ornementales exotiques exploitées dans les jardins urbains de la ville de Douala.

Méthodologie et Résultats : Des fiches d'enquête ont été soumises aux jardiniers dans Trois sites ont été délimités. Les espèces ornementales présentes dans les jardins de ces trois sites ont été recensées. Une méthode de notation des espèces recensées par attribution de pourcentage de citations a été mise sur pied. Les connaissances des jardiniers sur les espèces ornementales et les espèces invasives ont été évaluées. II ressort de cette étude que $64 \%$ des jardiniers ont des connaissances sur les espèces ornementales, mais leurs connaissances sur les espèces invasives restaient limitées (28\% d'avisés). Les 54 espèces ornementales recensées sont reparties en 44 genres et 28 familles. Les familles les plus représentées sont les Euphorbiaceae et les Araceae (16,7 \% des espèces recensées). Le type morphologique majoritaire est celui des arbrisseaux (54\%), les espèces recensées sont issues pour la plupart d'Asie et d'Amérique (81\%). Les modes de dissémination les plus fonctionnels sont la zoochorie et l'anémochorie $68 \%$. Le pourcentage de citation attribué à chaque espèce a permis d'établir 2 listes, une liste d'espèce en observation et une liste d'espèces potentiellement invasives.

Conclusion et Applications : Les études ultérieures permettront de confirmer ou d'infirmer le statut attribué aux espèces recensées.

Mots clés : espèces exotiques, invasion, biodiversité, horticulture, jardins, site

Objective: The objective of this study was to determine the invasive potential of exotic ornamental plants used in urban gardens in the city of Douala.

Methodology and Results: Three sites were chosen: The first was in Bonaberi, the second in Bonamoussadi and the third in Bonanjo with respectively 4, 3 and 3 urban gardens. Survey forms were submitted to the 
gardeners. Ornamental species in the gardens of the three sites were identified. A rating method identified by allocation percentage of citations species was established.. The knowledge of the gardeners on ornamental and invasive species was evaluated. As result of this study: $64 \%$ of the gardeners had some knowledge on ornamental species but their knowledge on the invasive species was limited (72\%). The 54 recorded ornamental species were divided into 44 genera and 28 families. The most represented family were the Euphorbiaceae and the Araceae (with $16.7 \%$ of recorded species). The most represented morphological type is the one of the shrubs (54\%). The recorded species most represented came from Asia and America ( $81 \%$ ). The most functional ways of dissemination are the zoochory and the anemochory $(68 \%)$. The percentage of citations assigned to every species established 2 lists, a list of observed species and a list of potentially invasive species.

Conclusion and Application findings: Further studies will confirm or invalidate the statute assigned to the recorded species.

Keywords: Alien species, invasion, biodiversity, Gardens, horticulture

\section{INTRODUCTION}

L'invasion des communautés végétales par les plantes introduites est considérée comme une des plus sérieuses menaces pour la biodiversité (Vitousek et al., 1997). Pour Usher (1988), il n'existe plus une zone naturelle protégée dans le monde, mis à part l'antarctique, n'abritant pas une plante introduite. La fragmentation ou la disparition des habitats, l'introduction d'espèces animales ou végétales exogènes et les dégâts liés à leur extension est un facteur d'importance majeure dans le mécanisme de la perte de diversité biologique (McNeely \& Strahm, 1997; Muller, 2004). Actuellement sous l'action de l'homme, la fréquence des invasions biologiques a considérablement augmenté et certaines espèces qui ne seraient jamais dispersées naturellement, ont été introduites dans différentes régions du monde (Hanfling \& Kollmann, 2002). Les invasions modernes ont pour cause première l'accroissement des échanges commerciaux internationaux ainsi que les nombreuses activités humaines provoquant volontairement ou involontairement le déplacement d'organismes vivants (agriculture, aquaculture, loisirs et transport) (Williamson, 1996 ; Kolar \& Lodge, 2001). Certaines plantes introduites pour une utilisation horticole par les pépinières, les jardins botaniques et les individus peuvent devenir invasives si elles abondent et entrent en compétition avec les plantes locales (Reichard \& White, 2001). Plusieurs plantes exotiques ornementales sont prédisposées à devenir envahissantes du fait de leur croissance rapide, de leur grande taille, de leur fertilité importante et de leur résistance aux maladies, autant de traits patiemment sélectionnés par les horticulteurs. Lorsqu'elles s'installent dans des milieux naturels, ces caractères leur permettent de développer des populations denses et de prendre le dessus sur les espèces indigènes (Branquart, 2011). De nombreuses plantes sont introduites hors de leur aire de répartition naturelle par l'homme pour des raisons alimentaires, fourragères, ornementales, ou encore mellifères ou médicinales. Parmi la multitude de plantes introduites, quelques espèces peuvent se répandre rapidement et avoir des impacts écologiques, économiques et/ou sanitaires négatifs ou jugés indésirables dans les milieux qu'elles colonisent avec vigueur. Ces espèces sont alors qualifiées d'espèces exotiques envahissantes (Muller, 2004). A partir de ces introductions volontaires ou accidentelles, certaines espèces se sont établies et ont prospérées au point d'échapper à tout contrôle et de se révéler nuisibles dans le nouvel environnement (Williamson, 1996; Richardson, 2001). Les scientifiques s'accordent sur le fait que ces espèces soient en majorité constituées d'espèces ornementales ; mais dans la région de Douala aucune étude n'a encore été menée dans le but de recenser ces espèces ornementales afin d'évaluer celles d'entre elles qui seraient invasives. L'objectif de cette étude est de 
recenser les espèces végétales ornementales exotiques (allochtones) exploitées dans les jardins urbains de la ville de Douala, et d'évaluer celles de ces espèces qui pourraient s'avérer invasives. Les objectifs spécifiques de cette étude sont: (1) recenser les espèces ornementales exploitées; (2)

\section{MATERIEL ET METHODES}

L'étude a été réalisée dans la ville de Douala, chef lieu de la région du Littoral. Les coordonnées géographiques de cette ville sont comprises entre $04^{\circ} 03^{\prime}-04^{\circ} 57^{\prime}$ de latitude Nord et $09^{\circ} 42^{\prime}-09^{\circ} 47$ de longitude Est avec une altitude moyenne de $13 \mathrm{~m}$. Elle occupe environ 18000 ha avec près de 2,5 millions d'habitants Les travaux se sont déroulés dans les jardins de fleurs situés dans trois grands sites de la ville de Douala : Bonaberi avec quatre jardins recensés (Bonassama, Texaco Fokou, Besseké et Bekoko), Bonamoussadi avec trois jardins recensés (Secteur Marie lumière, Rond point petit pays, Santa barbara) et Bonanjo avec trois jardins recensés (Marche des fleurs, PAD Bonanjo, Cimetière Njo Njo).

Les sites d'études ont été choisis en raison de la forte concentration des jardins de fleurs et de leur richesse spécifique et de leur diversité floristique en espèces ornementales.

\section{Méthodologie}

Collecte de Données : Les fiches d'enquêtes ont été conçues et soumises aux jardiniers lors des entretiens semi-structurés. Dans chacun des jardins, les informations ont été recueillies sur 30 plantes ornementales exploitées et des prises de vue de ces différentes plantes ont été effectuées.

Notation des espèces ornementales: Une méthode de notation des espèces ornementales recensées par

\section{RESULTATS}

Informations sur les jardiniers: Des 11 jardiniers enquêtés, 8 sont originaires de la région de l'ouest (70

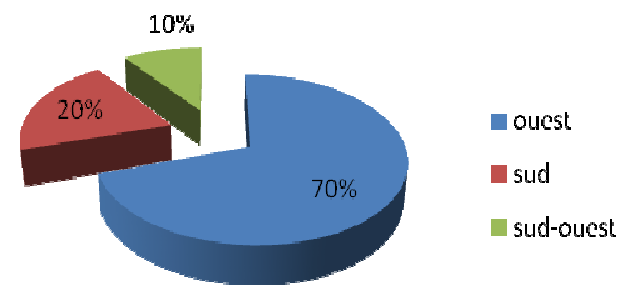

Figure 1 : Diagramme de fréquence des régions d'origine des enquêtés évaluer les connaissances des jardiniers; (3) déterminer les origines des espèces exploitées; (4) comparer la richesse spécifique des jardins urbains en fonction des sites étudiés; (5) et établir une liste d'espèces potentiellement invasives et une liste d'espèces en observation.

attribution d'un pourcentage de citation a été mise sur pied. Le pourcentage de citation a été obtenu en divisant le nombre de citation de chaque espèce par le nombre de citations total et en multipliant le résultat par 100. En fonction des pourcentages, deux listes d'espèces ont été établies dont une d'espèces en observation ( 0 à $50 \%$ ) et une d'espèces potentiellement invasives (51 à $95 \%$ ).

Analyses Statistiques : Le traitement de données a consisté à dépouiller les fiches d'enquêtes. La statistique descriptive a permis d'illustrer graphiquement les résultats et informations récoltées dans les jardins parcourues. L'analyse de variance (Anova) a permis d'évaluer la variation du nombre de citations d'espèces en fonction des sites parcourues et les tests de corrélation ont permis de déterminer les relations entre les sites. L'analyse statistique visant les paramètres suffisamment discriminants entre les jardins et les sites a permis de les regrouper en un nombre restreint de classes homogènes. Les regroupements suivant le degré de similarité (coefficient de corrélation de Pearson) ont été effectués grâce à une classification ascendante hiérarchique (Dendrogramme). Les logiciels de statistiques utilisés sont: Excel 2007, pour la statistique descriptive; Graphpadprism 5, pour l'analyse de variance ; R 3.0.1, pour le test de Corrélation et XL STAT 2013, pour la classification ascendante hiérarchique.

$\%), 2$ de la région du sud (20\%) et enfin 1 seul de la région du sud-ouest (10\%) (Figure 1).

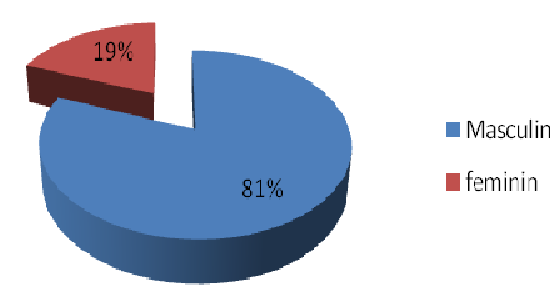

Figure 2 : Diagramme de sexe des enquêtés. 
Tchatat et al. J. Appl. Biosci. 2014. Potentiel invasif des espèces végétales exotiques ornementales des jardins de fleurs de la ville de Douala

Les personnes enquêtées comptent 9 hommes ( $81 \%)$, et 2 femmes (19\%) (Figure 2). Parmi les jardiniers

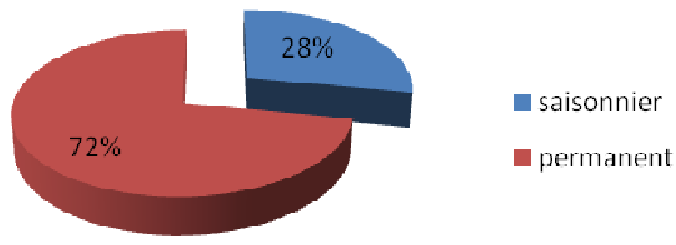

Figure 3: Diagramme de fréquence des statuts des différents enauêtés.

Quatre des jardiniers enquêtés sont jeunes ( 18 à 29 ans), $36 \%, 5$ sont adultes ( 30 à 49 ans), $46 \%$ et 2 sont vieux (50 ans et plus), $18 \%$ (Figure 4).

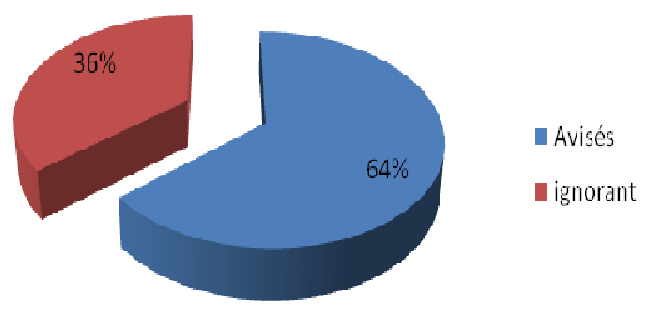

Figure 5 : Diagramme des connaissances des enquêtés sur les espèces ornementales.

Les jardiniers enquêtés comptent 8 ignorants au sujet des espèces invasives (73\%) et 3 Avisés (28\%). (Figure 6)

Recensement des espèces ornementales : L'inventaire des espèces ornementales a permis de recenser 54 enquêtés, 8 sont permanents ( $72 \%$ ) et 3 saisonniers ( 28 $\%$ ) (Figure 3).

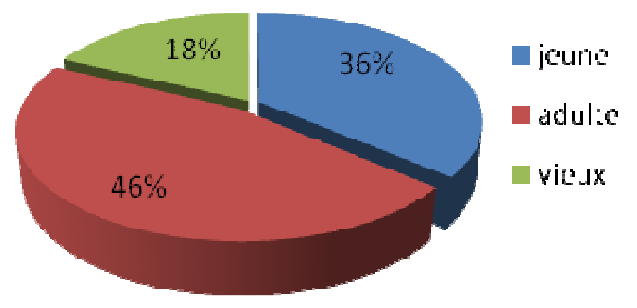

Figure 4: Diagramme circulaire d'âge des enquêtés.

Évaluation des connaissances des jardiniers enquêtés : Parmi les jardiniers enquêtés, 7 ont des connaissances sur les espèces ornementales (64 \%), et 4 en sont ignorants (36 \%) (Figure 5).

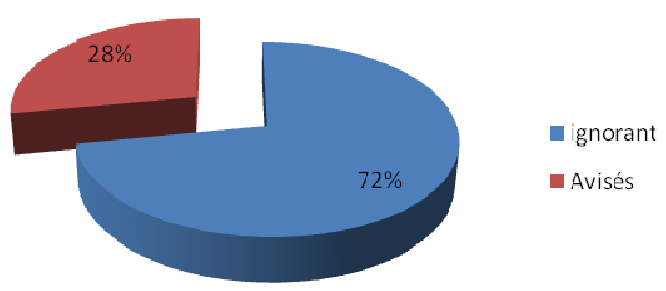

Figure 6: Diagramme de fréquences concernant les connaissances des jardiniers sur les Invasives.

espèces, reparties en 44 genres et 28 familles. Les familles des Euphorbiacées et des Aracées sont les plus représentées avec respectivement 5 et 4 espèces (soient $9,3 \%$ et $7,4 \%$ ) (Figure 7 ).

\section{Frequence des familles}

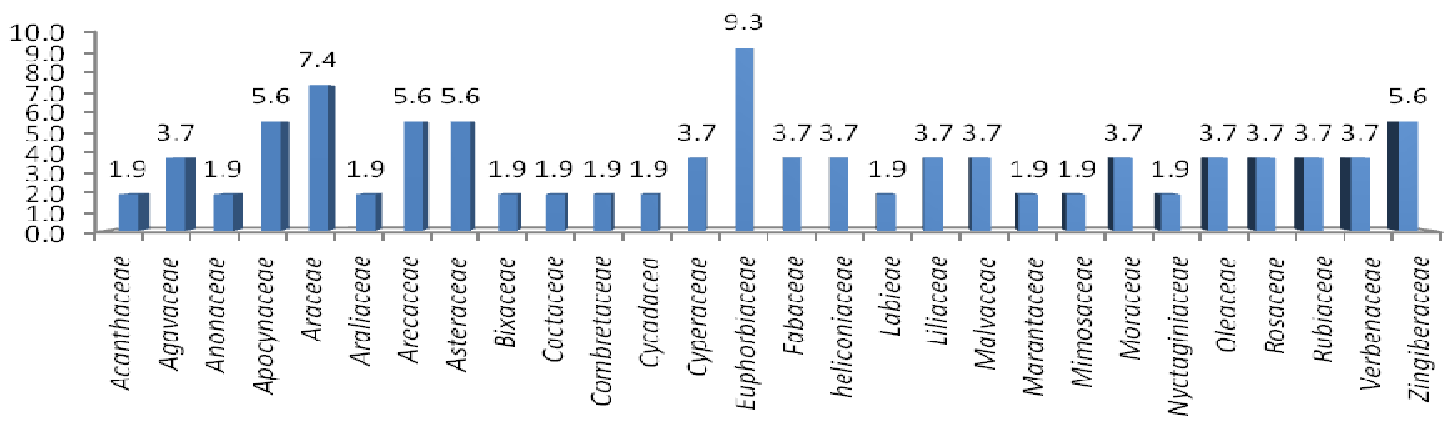


Figure 7 : Histogramme concernant les Fréquences des familles des espèces recensées.

Les espèces recensées sont reparties en trois types morphologiques : 5 arbres (9\%), 20 arbustes (37\%) et 29 arbrisseaux (54\%) (Figure 8).

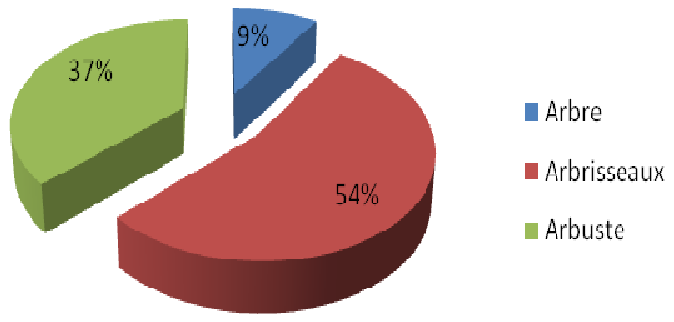

Figure 8 : Fréquence des types morphologiques des espèces recensées.
Origine des espèces ornementales exotiques : Parmi les espèces exotiques recensées, 5 sont originaires d'Afrique (9\%), 6 sont d'Europe (10\%), 20 sont d'Asie (38\%) et enfin 23 d'Amérique. (43\%) (Figure 9).

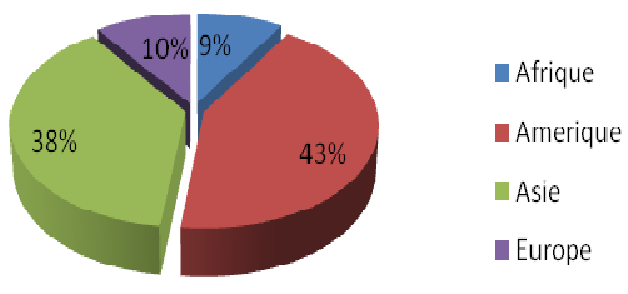

Figure 9 : Diagramme circulaire des origines des espèces ornementales recensées.

\section{Pourcentage mode de dissemination}

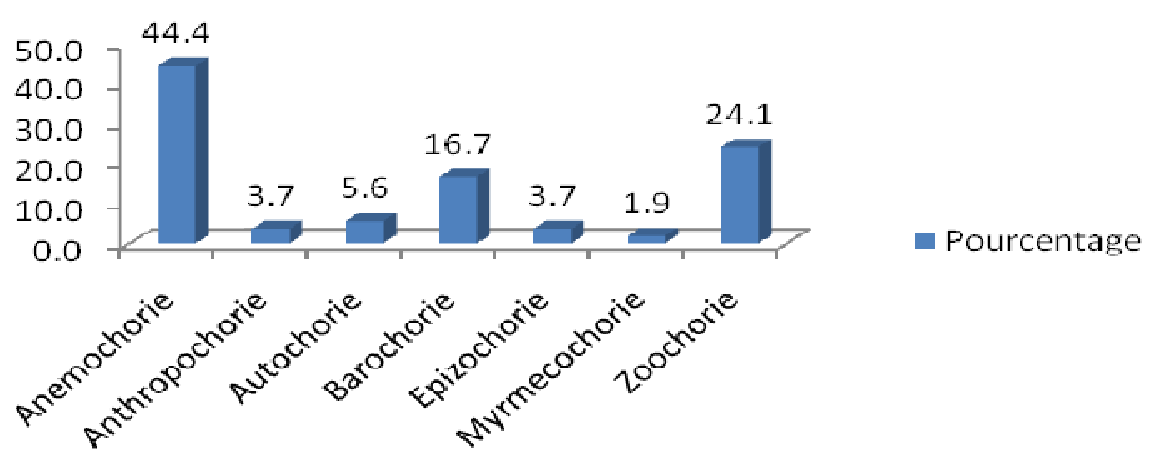

Figure 10 : Histogramme des fréquences des modes de dissémination des espèces recensées.

Vingt quatre des espèces ornementales recensées ont pour mode de dissémination l'anémochorie $(44,4 \%), 2$ ont pour mode l'anthropochorie (3,7 \%), 3 l'autochorie (5,6\%), 9 la barochorie (16,7\%), 2 l'epizochorie (3,7\%), 1 la myrmecochorie $(1,9 \%)$ et enfin 13 ont pour mode de dissémination la zoochorie $(24,1 \%)$ (Figure 10).
Citations des familles: Cinq familles d'espèces ont été reconnues comme les plus citées : Les Euphorbiaceae (34 citations), les Apocynaceae (21), les Araceae (19), les Arecaceae et les Rubiaceae (15 citations chacune) (Figure 11). 


\section{total citation famille}

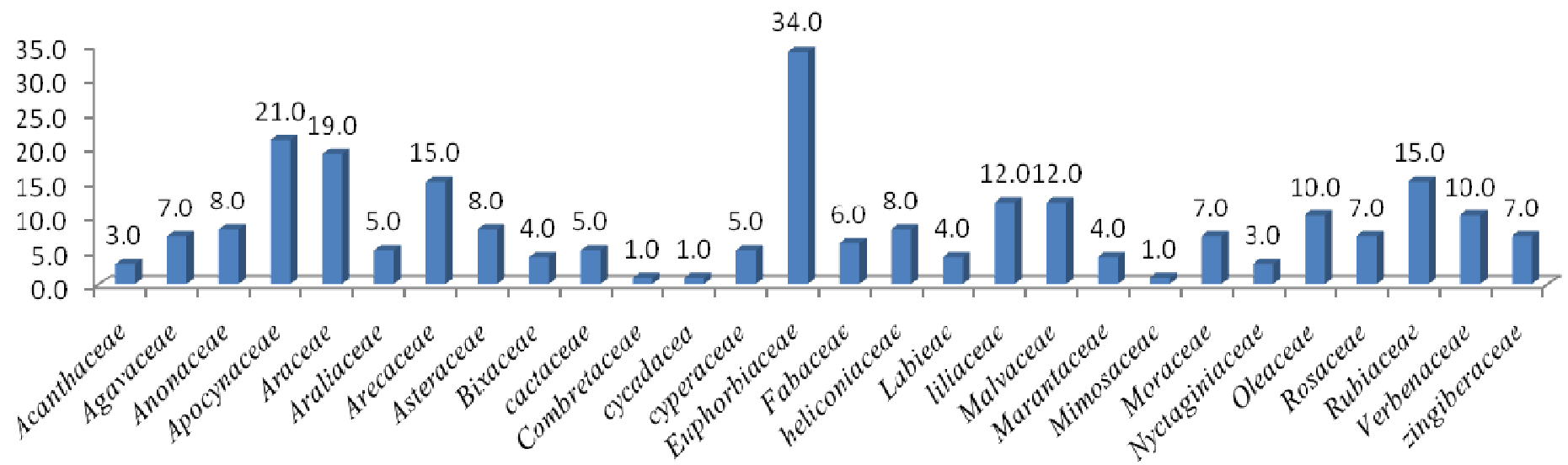

Figure 11 : dans les trois sites sont : Breynia nivosa (W.Bull) L., Histogramme des totaux de citations des familles d'espèces ornementales recensées.

Citations d'espèces dans les trois sites parcourus: Les espèces les plus citées Hibiscus rosa-sinensis L., Ixora coccinea L. et Ligustrum coriaceum avec un total de 9 citations chacune (Figure 12).

\section{Total de citation d'espèces}

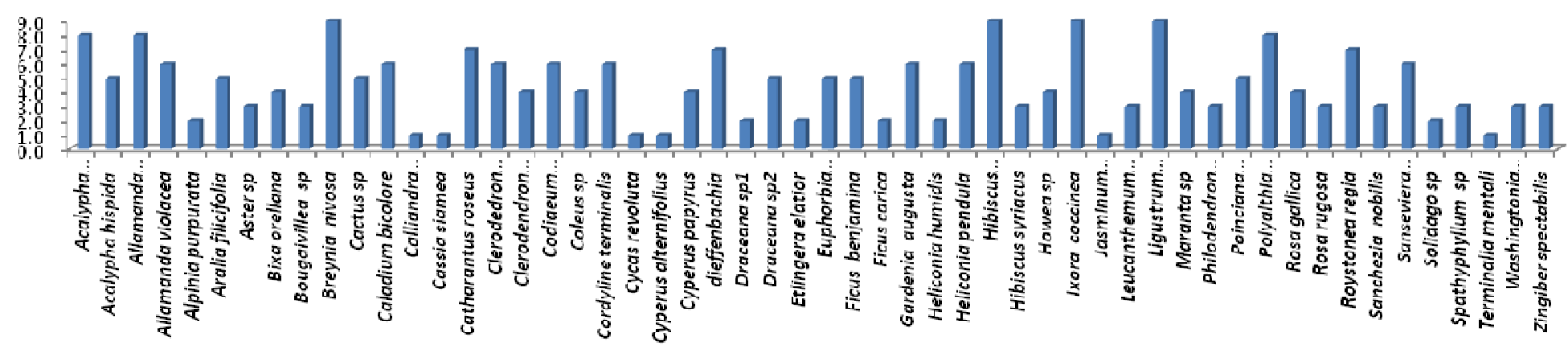


Figure 12 : Histogramme des totaux de citation des espèces ornementales recensées dans les trois sites.

Citations d'espèces du site 1 (Bonaberi) : Les espèces ornementales les plus citées dans le site 1 sont : Breynia nivosa (W. Bull) L., Ixora coccinea L. et Ligustrum coriaceum avec 4 citations chacune (Figure 13).

Total de citations d'espèces

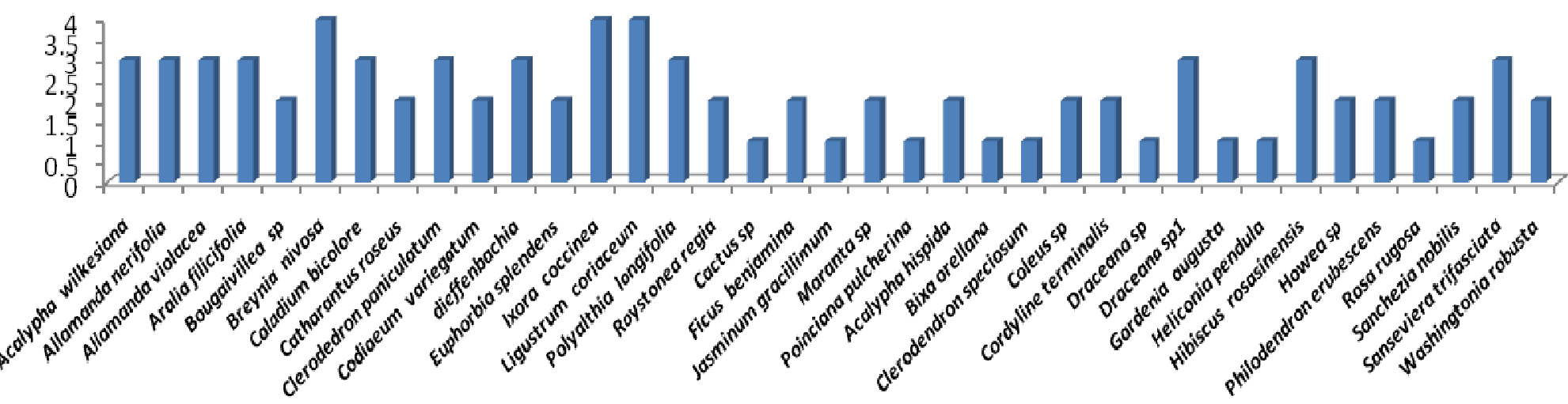

Figure 13 : Histogramme du nombre de citation d'espèces ornementales du site 1 (Bonaberi).

Citations d'espèces du site 2 (Bonamoussadi): Les espèces ornementales les plus citées dans le site 2 sont : Acalypha hispida Burm.f, Acalypha wilkesiana Burm.f, Breynia nivosa (W. Bull) L., Caladium bicolore Aiton(Vent), Catharantus roseus (L.) G. Don, Clerodendron panniculatum Juss., Codiaeum variegatum (L.)
A. Juss., Dieffenbachia Schott, Euphorbia splendens L., Hibiscus rosa-sinensis L., Ixora coccinea L., Ligustrum coriaceum L., Polyalthia longifolia Sonn., Rosa rugosa L. et Roystonea regia (Kunth) O. F. Cook avec 3 citations chacune (Figure 14). 
Total de citations d'espèces

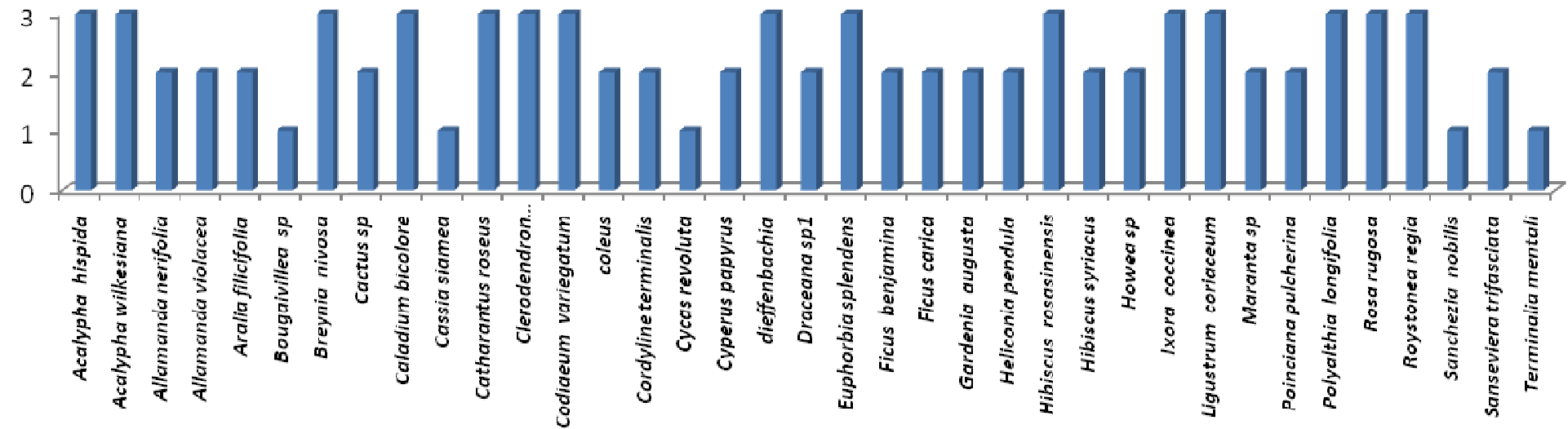

Figure14 : Histogramme du nombre de citation d'espèces ornementales du site 2 (Bonamoussadi).

Citations d'espèces du site 3 (Bonanjo): Les espèces ornementales les plus citées dans le site 3 sont : Acalypha hispida Burm. f, Acalypha wilkesiana Burm.

f, Allamanda cathartica L., Heliconia humidis L., Heliconia pendula L., Hibiscus rosa-sinensis L., Ixora coccinea L., Leucanthemum vulgare Lam, Ligustrum coriaceum L. et Zingiber spectabilis Griff, avec 3 citations chacune (Figure 14). 
Total de citations d'espèces

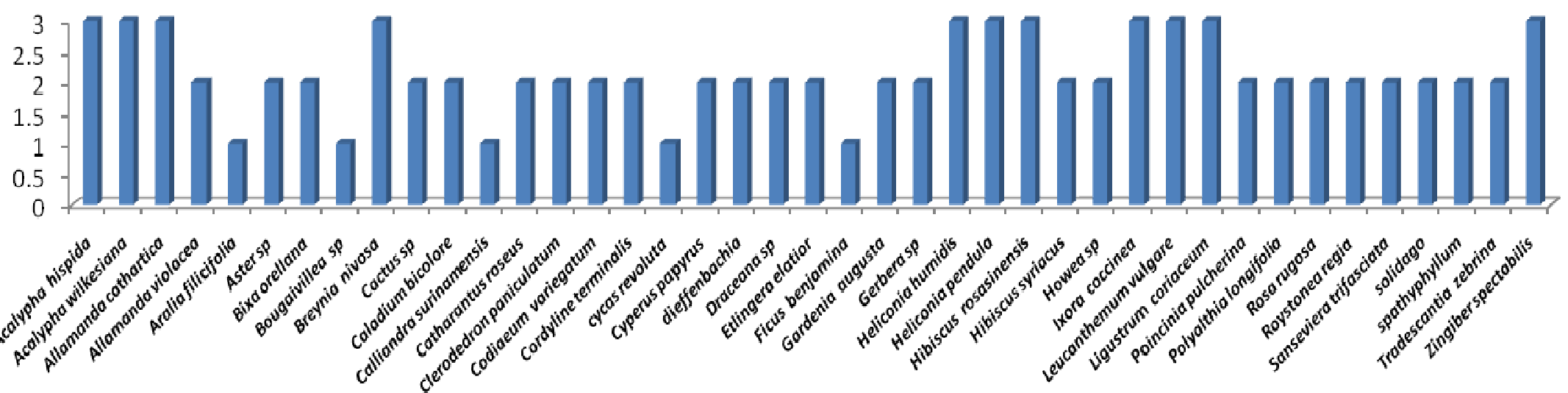

Figure 14 : Histogramme du nombre de citation d'espèces ornementales du site 3 (Bonanjo). 
Tchatat et al. J. Appl. Biosci. 2014. Potentiel invasif des espèces végétales exotiques ornementales des jardins de fleurs de la ville de Douala

Parmi les 54 espèces ornementales recensées 28 sont communes aux trois sites, 9 sont communes aux sites 1 et 2, deux sont communes aux sites 1 et 3 et enfin 3 autres sont communes aux sites 2 et 3 . Une espèce citée est exclusive au site 1 , une autre est exclusive au site 2 et 10 sont exclusives au site 3 . Les espèces communes aux trois sites sont : Allamanda violacea L., Clerodendron paniculatum L., Clerodendron speciosum L., Cactus sp L., Codiaeum variegatum (L.)A Juss, Cordyline terminalis L., Gardenia augusta J. Elis, Heliconia pendula L., Sanseviera trifasciata Prain, Catharantus roseus (L.) G. Don, Dieffenbachia sp. Schott, Roystonea regia (Kunth) O. F. Cook, Acalypha wilkesiana Burm.f, Allamanda cathartica L., Polyalthia longifolia Sonn., Breynia nivosa (W. Bull), Hibiscus rosa-sinensis L., Hibiscus syriacus L., Ixora coccinea L., Ligustrum coriaceum L., Aralia filicifolia L., Bixa Orellana L. Draceaena sp. 2 Hort. Sander, Ficus Benjamina L., Rosa Gallica L., Rosa rugosa L., Maranta $\mathrm{sp}$. Les espèces communes aux sites 1 et 2 sont: Acalypha hispida Burm.f, Caladium bicolore (Aiton) Vent, Maranta sp., Terminalia mentali, Bougainvillea sp. Comm. Ex Juss., Philodendron erubescens K. Koch \& Augustin, Sanchezia nobilis, Coleus sp., Euphorbia splendes P. Miler. Les espèces communes aux sites 1 et 3 sont : Draceana sp. 1 Hort sander, Washimgtonia robusta H.Wendl. Les espèces communes aux sites 2 et 3 sont : Cyperus alternifolius L., Cyperus papyrus L. Ficus carica L. L'espèce exclusive du site 1 est Jasminum gracillinum, celle exclusive du site 2 est Cassia siamea L. et celles exclusives du site 3 sont Alpinia purpurata Vieill, Astersp. L., Calliandra surinamensis, Cycas revoluta L., Etlingera elatior (jack) R. M, Heliconia humidis L., Leucanthemum vulgare Lam, Solidago sp. L Woldst \& Kitt, Spathyphyllum sp. Liebm., Zingiber spectabilis Griff.
Nombre de citations d'espèces ornementales en fonction des sites: La probabilité d'acceptation des hypothèses est inferieure à 0,005 ( $p$-valeur $=0,0012$ ), indiquant une significativité à 1 pour mille et permet de conclure qu'il y a $99 \%$ de chances que le nombre de citation des espèces ornementales varient en fonction des sites parcourues. La corrélation est significative entre le nombre de citations d'espèces ornementales des sites 1 et 2 ( $p$-valeur $=2.88$ e-13) et un degré de corrélation de 0,80 . Par contre, les corrélations entre le nombre de citations d'espèces dans les sites 1 et 3 et les sites 2 et 3 ne pas été significatives (Figure 15).

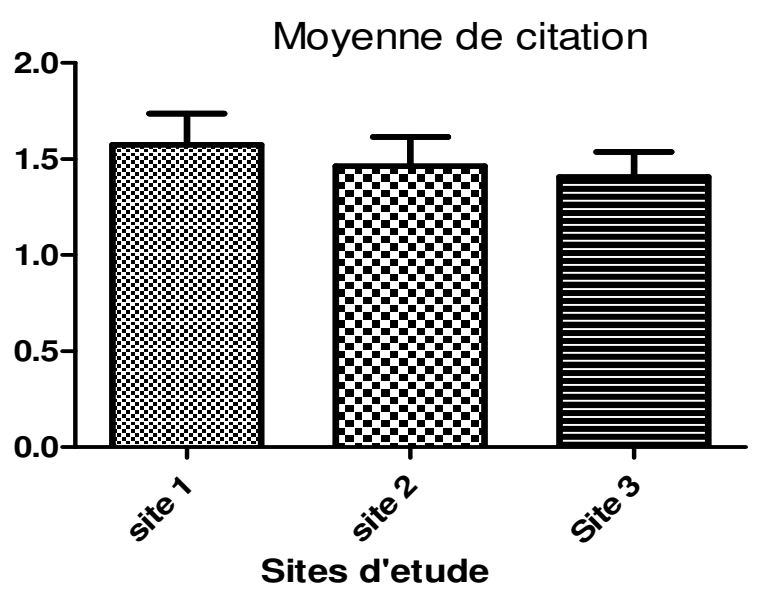

Figure 15: Histogramme des moyennes de citations d'espèces ornementales dans les trois sites.

Classification ascendante hiérarchiques des sites recensés: L'analyse du dendrogramme indique une forte affinité entre les sites 1 et 2 avec un coefficient de similarité de Pearson de 0,927 (Figure 16). 


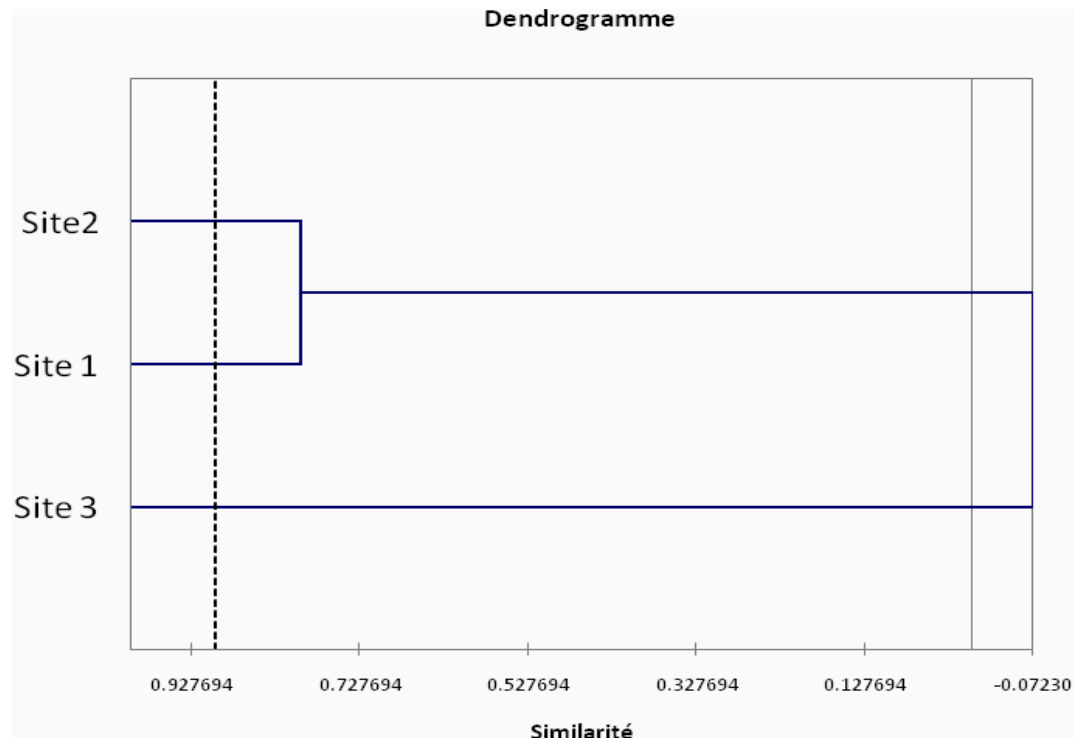

Site 1 : Bonaberi , Site 2 : Bonamoussadi, Site 3 : Bonanjo

Figure 16 : Dendrogramme des sites en fonction des espèces ornementales citées.

Classification ascendante hiérarchique des jardins recensés: L'analyse du dendrogramme révèle des affinités entre jardins de même site : jardins 8 et 9 du site 3 , jardins 2 et 3 du site 1 et jardins 5 et 6 du site 2 . Le dendrogramme montre aussi des affinités entre jardins de sites différents : jardins 7 et 10 respectivement des sites 2 et 3 et les jardins $1,2,3$ et 5,6 respectivement des sites 1 et 2 (Figure 17).

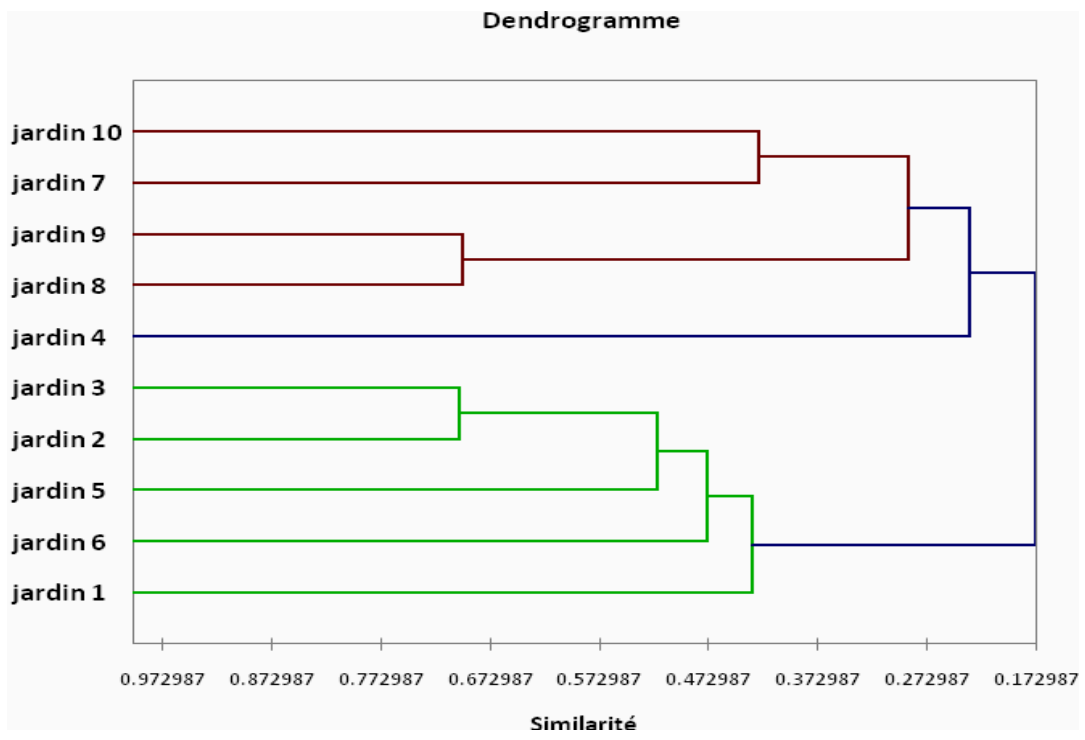

Jardin 1 : Texaco Fokou, Jardin 2 : Bonassama, Jardin 3 : Besseke, Jardin 4 : Bekoko, Jardin 5 : Rond point petit pays Makepe, Jardin 6 : Secteur marie lumière, Jardin $7:$ Bonamoussadi, Jardin 8 : Marché des fleurs Bonanjo, Jardin 9 : PAD Bonanjo, Jardin 10 : Cimetière Njo Njo

Site 1 : jardins $1,2,3$ et $4 . \quad$ Site $2:$ Jardins 5,6 et 7 . Site $3:$ Jardins 8,9 et 1

Figure 17 : Dendrogramme des jardins en fonction des espèces ornementales citées. 
Tchatat et al. J. Appl. Biosci. 2014. Potentiel invasif des espèces végétales exotiques ornementales des jardins de fleurs de la ville de Douala

Deux listes d'espèces se dégagent du nombre des citations : les espèces en observation ( 0 à $50 \%$ ) et les espèces potentiellement invasives (51 à $95 \%$ ) (Tableau 1).

Tableau 1 : Citations des espèces ornementales recensées.

\begin{tabular}{|c|c|c|c|c|}
\hline Noms scientifiques & Noms communs & Familles & $\begin{array}{lr}\text { Total } & \text { de } \\
\text { citation } / 10\end{array}$ & $\begin{array}{l}\text { Pourcentage de } \\
\text { citation } \%\end{array}$ \\
\hline Acalypha wilkesiana & queue d'écureuil & Euphorbiaceae & 8 & 80 \\
\hline Acalypha hispida & acalypha panache & Euphorbiaceae & 6 & 60 \\
\hline Allamanda cathartica & allamanda & Apocynaceae & 8 & 80 \\
\hline Allamanda violacea & allamanda violet & Apocynaceae & 6 & 60 \\
\hline Alpinia purpurata & alpine & zingiberaceae & 2 & 20 \\
\hline Aralia filicifolia & aralia & Araliaceae & 5 & 50 \\
\hline Aster sp. & aster & Asteraceae & 3 & 30 \\
\hline Bixa orellana & roucou & Bixaceae & 4 & 40 \\
\hline Bougaivillea sp. & bougainvillea & Nyctaginiaceae & 3 & 30 \\
\hline Breynia nivosa & frigelia & Euphorbiaceae & 9 & 90 \\
\hline Cactus sp. & cactus & Cactaceae & 5 & 50 \\
\hline Caladium bicolore & caladium & Araceae & 6 & 60 \\
\hline Calliandra surinamensis & caliandra & Mimosaceae & 1 & 10 \\
\hline Cassia siamea & cassia jaune & Fabaceae & 1 & 10 \\
\hline Catharantus roseus & pervenche & Apocynaceae & 7 & 70 \\
\hline Clerodedron paniculatum & clerodendron & Verbenaceae & 6 & 60 \\
\hline Clerodendron speciosum & clerodendron & Verbenaceae & 4 & 40 \\
\hline Codiaeum variegatum & croton & Euphorbiaceae & 6 & 60 \\
\hline Coleus sp. & coleus & Labiae & 4 & 40 \\
\hline Cordyline terminalis & cordyline & liliaceae & 6 & 60 \\
\hline Cycas revoluta & rameau & Cycadacea & 1 & 10 \\
\hline Cyperus alternifolius & cyperus & Cyperaceae & 1 & 10 \\
\hline Cyperus papyrus & cyperus & Cyperaceae & 4 & 40 \\
\hline Dieffenbachia & compacta & Araceae & 7 & 70 \\
\hline Draceana sp. 1 & $\begin{array}{l}\text { arbre de la paix } \\
\text { panache }\end{array}$ & Agavaceae & 2 & 20 \\
\hline Draceana sp. 2 & arbre de la paix & Agavaceae & 5 & 50 \\
\hline Etlingera elatior & rose de porcelaine & Zingiberaceae & 2 & 20 \\
\hline Euphorbia splendens & epine du christ & Euphorbiaceae & 5 & 50 \\
\hline Ficus benjamina & ficus & Moraceae & 5 & 50 \\
\hline Ficus carica & ficus panache & Moraceae & 2 & 20 \\
\hline Gardenia augusta & gardenia & Rubiaceae & 6 & 60 \\
\hline Heliconia humidis & heliconia & Heliconiaceae & 2 & 20 \\
\hline Heliconia pendula & heliconia & Heliconiaceae & 6 & 60 \\
\hline Hibiscus rosasinensis & hibiscus & Malvaceae & 9 & 90 \\
\hline Hibiscus syriacus & althea & Malvaceae & 3 & 30 \\
\hline Howea sp. & palmier metisse & Arecaceae & 4 & 40 \\
\hline Ixora coccinea & Ixora & Rubiaceae & 9 & 90 \\
\hline Jasminum gracillimum & Jasmin & Oleaceae & 1 & 10 \\
\hline Leucanthemum vulgare & marguerite & Asteraceae & 3 & 30 \\
\hline Ligustrum coriaceum & troene panache & Oleaceae & 9 & 90 \\
\hline
\end{tabular}


Tchatat et al. J. Appl. Biosci. 2014. Potentiel invasif des espèces végétales exotiques ornementales des jardins de fleurs de la ville de Douala

\begin{tabular}{|l|l|l|l|l|}
\hline Maranta sp. & maranta & Marantaceae & 4 & 40 \\
\hline Philodendron erubescens & philodendron & Araceae & 3 & 30 \\
\hline Poinciana pulcherina & orgueil de chine & Fabaceae & 5 & 50 \\
\hline Polyalthia longifolia & sol pleureur & Anonaceae & 8 & 80 \\
\hline Rosa rugosa & rosier & Rosaceae & 4 & 40 \\
\hline Rosa gallica & roses & Rosaceae & 3 & 30 \\
\hline Roystonea regia & palmier royal & Arecaceae & 7 & 70 \\
\hline Sanchezia nobilis & hortancia & Acanthaceae & 3 & 30 \\
\hline Sanseviera trifasciata & sanseviere & Liliaceae & 6 & 60 \\
\hline Solidago sp. & aster solidago & Asteraceae & 2 & 20 \\
\hline Spathyphyllum sp. & arbre a camphre & Araceae & 3 & 30 \\
\hline Terminalia mentali & terminalia & Combretaceae & 1 & 10 \\
\hline Washingtonia robusta & washingtonia & Arecaceae & 4 & 40 \\
\hline
\end{tabular}

\section{DISCUSSION}

Les jardiniers enquêtés sont en majorité des hommes ( 81 $\%)$, jeunes et adultes (82\%), car l'horticulture est une activité contraignante et exigeante sur le plan physique (CHSCT, 2012). La plupart des jardiniers ont des connaissances sur les espèces ornementales (64\%). Toutefois leurs connaissances sur les espèces invasives restent limitées (28 \% d'avisées). Les espèces ornementales recensées sont en majeur partie des arbrisseaux et des arbustes (91\%). Selon les jardiniers ces types morphologiques sont les plus prisées par les acheteurs en raison de leur pérennité. Les espèces recensées sont surtout originaires d'Amérique et d'Asie (respectivement $43 \%$ et $38 \%$ ). Ce constat est en accord avec ceux Magnanon et al. (2008) qui révèlent que sur 56 plantes exotiques invasives avérées et potentielles recensées dans le nord-ouest de la France, $55 \%$ sont originaires du continent américain et $21,5 \%$ du continent asiatique. Selon Vahrameev et Nobiliaux (2013), le facteur justifiant la prédominance des espèces américaines et asiatiques est leur origine tropicale. Conséquemment, elles trouveraient dans leurs milieux d'implantation des conditions environnementales et climatiques semblables à leur d'origine. L'anémochorie et la zoochorie $(68,5 \%)$ sont les modes de dissémination les plus fonctionnels, car favorable à la prolifération et à l'invasion (Filippi \& Aronson, 2010). Ces modes de dissémination à longue distance augmentent le risque d'échappement des jardins et d'accessibilité éventuelleà d'autres milieux où elles peuvent proliférer et devenir invasives. Mack et al. (2005) corroborent dans ce sens en affirmant que les espèces qui présentent un risque potentiel d'invasion sont celles qui ont des capacités de dispersion efficaces, augmentant leurs chances d'échappement des jardins. De plus, certaines espèces ornementales recensées sont dotées de grandes fleurs colorées et d'inflorescences denses qui présentent une corolle ouverte et produisent un nectar et du pollen facilement accessibles par les insectes pollinisateurs aptes à visiter une grande diversité de fleurs (Branquart et al., 2011).

Les familles les plus représentées sont celles des Euphorbiaceae et des Araceae (respectivement 9,3\% et $7,4 \%$ ) suivi des Arecaceae 5,6\%. La prédominance de ces familles a déjà été constatée par Fleriag (2009) au cours d'un recensement de 275 espèces ornementales avec la prédominance des Arecaceae $19 \%$, des Araceae $18 \%$ et des Euphorbiaceae $17 \%$. Le test Anova a montré une variation du nombre des citations des espèces ornementales en fonctions des sites et donc forcément en fonction des jardins parcourus. Les tests de corrélation ont permis de rapprocher les sites 1 et 2 qui ont en commun plusieurs espèces ornementales recensées. Le site 3 (Bonanjo) est un quartier administratif et résidentiel, les espèces ornementales retrouvées dans les jardins de ce site sont celles prisées par une classe bourgeoise très attachée à l'esthétique et à l'exotisme. Par contre les espèces ornementales des sites 1 et 2 (Bonaberi et Bonamoussadi) sont courantes. Luginbuhl (2004) aborde dans ce sens en affirmant que depuis le 19 siècle l'usage des plantes ornementales en ville est resté pendant longtemps l'apanage des classes aisées qui cherchaient dans cette pratique un moyen de se démarquer de la paysannerie et des jardins familiaux et périurbains où abondent les espèces vulgaires (sites 1 et 2). L'affinité entre certains des jardins des sites 2 et 3 , notamment les jardins 7 et 10, peut s'expliquer par leur gestion modernisée par des professionnels de l'horticulture, d'où la gamme importante d'espèces 
exotiques ornementales exploitées. Les espèces en observation sont celles reconnues comme moins citées dans les jardins, néanmoins, certaines de ces espèces sont déjà considérées comme invasives ailleurs il s'agit de : Aster sp. Voss., Solidago sp. L Woldst Kitt, Cyperus papyrus L., Cyperus alternifolius L., respectivement

\section{CONCLUSION}

L'étude des espèces végétales ornementales de la région de Douala a été réalisée dans le but d'analyser le potentiel invasif des espèces présentes dans les jardins des différents sites d'étude. Les acteurs de ce secteur sont en majorité des hommes jeunes et adultes originaires de la région de l'Ouest ayant une activité permanente, avec des connaissances rudimentaires sur les espèces ornementales et invasives. L'étude a permis de recenser 54 espèces ornementales qui sont en majorité des arbustes et arbrisseaux originaires d'Asie et

\section{REFERENCES}

Alter IAS. 2011. Enquête socio économique sur les plantes invasives en Belgique.10 p

Branquart E, 2011. Alert black and watch list of invasive species in Belgium. Harmonia version 1.2, Belgian Forum on invasive species.

CHSCT, 2012. Comite d'Hygiène de sécurité et des conditions de travail. Prévention des risques professionnels de l'horticulture et du maraichage. France, aout 2012

Filippi O, Aronson J, 2010. Plantes invasives en région méditerranéenne: quelles restrictions d'utilisation à préconiser pour les jardins et les espaces verts? Ecologia Mediterranea 36 (2): 31-54.

Fleriag L, 2009. Participation aux travaux préparatoires à l'établissement de la liste des espèces exogènes potentiellement invasives à interdire a l'importation. Mémoire de Master 1ere annee. Universite de Perpignan. Option Biodiversité et développement durable. 56p.

Hanfling B, Kollmann J, 2002. An evolutionary perspective of biological invasions. Trends in Ecology and Evolution 17 : 545-546.

Luginbuhl Y, 2004. Un monde au balcon, la nature dans le quartier. Projet Paris, pp. 96-98.

Kolar CS, Lodge DM, 2001. Progress in invasion biology: predicting invaders. Trends in Ecology and Evolution 16: 199-204. invasives en France, Belgique et au Nigeria (Muller, 2004 ; Alter, 2010 ; UICN, 2004, 2013). Les espèces potentiellement invasives nécessitent une attention très particulière dans les travaux ultérieures afin d'établir des stratégies de lutte et de gestion durable.

d'Amérique. Les familles regroupant le plus d'espèces sont celles des Euphorbiaceae et des Araceae et les modes de dissémination les plus fonctionnels sont la Zoochorie et l'Anémochorie. Une variation des espèces citées en fonction des jardins et des sites d'étude a été observée. Le pourcentage des citations a permis d'établir deux listes, une liste d'espèces en observation (entre 0 et $50 \%$ ) et une liste d'espèces potentiellement invasives (entre 51 et $95 \%$ ).

Mack RN. 2005 predicting the identity of plant invaders: future contributions from horticulture. Hortscience 40 (5): 1168-1174.

MacNeely J, strahm W. 1997, L'U.I.C.N. et les espèces étrangères envahissantes : un cadre d'action. Conservation de la vitalité et de la diversité. U.I.C.N. (Editor), Congrès mondial sur la conservation, Ottawa, pp. 3-10.

Magnanon S, Geslin J, Lacroix P, Zambettakis C, 2008. Examen du statut d'indigénat et du caractère invasif des plantes vasculaires de BasseNormandie, Bretagne et Pays de la Loire .Proposition d'une première liste d'espèces invasives et potentiellement invasives pour ces regions. Bulletin du conservatoire national de Brest : 90-96.

Muller S, 2004. Plantes invasives en France, collection patrimoines de naturels, Vol. 62, publication scientifiques du Muséum national d'histoire naturelle, Paris. 168p.

Reichard SH, White P. 2001. Horticulture as a pathway for invasive plant introductions in the United States. Bioscience 51: 103-113.

Richardson DM. 2001. Plant invasions. In: Encyclopedia of biodiversity Levin S (editor), Academic press, San Diego, pp. 677-688.

UICN, 2004. Prévention et gestion des espèces étrangères invasives.

UICN, 2013. Plantes invasives affectant les aires protégées. 
Tchatat et al. J. Appl. Biosci. 2014. Potentiel invasif des espèces végétales exotiques ornementales des jardins de fleurs de la ville de Douala

Usher MB, Kruger FJ, Macdonald IAW, Loope LL Brockie, RE, 1988. The ecology of biological invasions into nature reserves: an introduction. Biol. Conserv: 44: 1-8.

Vahrameev P, Nobiliaux S. 2013. .Liste d'espèces végétales invasives de la région Centre, version 3. Conservatoire botanique national du bassin parisien, Délégation du Centre. 41p.
Vitousek PM, D'antonio CM, Loope LL, Rejmanek M, Westbrooks R. 1997. Introduced species: A significant component of human-caused global change. New Zealand Journal of Ecology 21: 116.

Williamson M, 1996. Biological invasions. Chapman and Hall, London. 94p. 